\title{
Epidemiological Survey of Visceral Leishmaniasis: An Evidence Based Report
}

\author{
${ }^{1}$ Mohammad Arif Khan, ${ }^{1}$ Ravada Vinay Kumar, \\ ${ }^{2}$ Krishna Pandey, ${ }^{1}$ Krishna Murti and ${ }^{1}$ Ashok Kumar Gupta \\ ${ }^{1}$ Department of Pharmacy Practice, NIPER, Hajipur (Bihar), India \\ ${ }^{2}$ Department of Medicine, Rajendra Memorial Research Institute of Medical Sciences, Patna (Bihar) India
}

Received 2013-06-20, Revised 2013-07-28; Accepted 2013-07-29

\begin{abstract}
The aim of this study was to find out the epidemiology of visceral leishmaniasis among individuals of different age group and sex living in rural communities of Bihar, India. A retrospective study was conducted on 133 patients with a history of prolong fever and weight loss who was admitted at Rajendra Memorial Research Institute Of Medical Sciences (RMRIMS), Patna, India during the year 2013 (from January 2013 to April 2013). It was found that majority of the cases $(64.67 \%)$ cases were of adults aged between $15-45$ years of age, $21.80 \%$ cases were of children below 14 years of age whereas $13.5 \%$ cases belong to patients of age more than 45 years of age including elderly patients. Leishmaniasis is a life threatening disease affecting poorest of the poor.
\end{abstract}

Keywords: Epidemiology, Visceral Leishmaniasis, Incidence, Sex Ratio

\section{INTRODUCTION}

The term Visceral Leishmaniasis also known as 'kala azar 'black fever' or 'dumdum' fever, originates from India, which refers to the hyperpigmentation of skin during the course of the disease. Every year estimated 2 million new cases occur, of which 50,000 cases are of Visceral Leishmaniasis (VL) whereas 1,50000 cases are of Cutaneous Leishmaniasis. Every year, the estimate for incidence and prevalence of kalaazar over the world is 0.5 and 2.5 million, respectively, with an overall case-fatality rate of $10 \%$ and assuming that virtually all deaths are from VL, estimate of 20,000 to 40,000 leishmaniasis deaths occur per year, in line with previous WHO estimates (WHO, 2008). VL is the most severe form of leishmaniasis, second largest parasitic killer in the world (after malaria), responsible for an estimated 500,000 infections each year world wide. An estimated 500,000 new VL cases and 60,000 deaths from VL occur each year (Ready, 2008). From
1987 through 2011, a total of 6,70,897 kala-azar cases were reported officially from Bihar only, the data were collected by the National Institute of Communicable Disease (NICD) and Directorate General of Health Services, Government of India at the block level (public health centres). Leishmaniasis consists of four main clinical syndrome-Cutaneous leishmaniasis (caused by L.donovani), Muco-cutaneous leishmaniasis (caused by L.braziliensis), Dermal leishmaniasis (caused by L.tropica) and Post Kala-azar Dermal Leishmaniasis (PKDL) which can occur as a sequence to kala-azar, which was first described by Brahmchari. The clinical symptoms of VL include prolong fever, fatigue, weight loss, bleeding tendency and an enlarged spleen and liver. Leishmaniasis consists of a complex of vectorborne diseases caused by more than 20 species of the protozoan genus Leishmania and is transmitted by sand fly vectors (WHO, 2010). Two species of leishmaniasis are known to give rise to visceral form of the disease. Species commonly found in East Africa and Indian Hajipur (Bihar), India Tel: +91-9470813689 
subcontinent is L.donovani and that found in Europe, North Africa and Latin America is L. infantum, also known as L.chagasi Natural transmission may be zoonotic or anthroponotic by the bite of a phlebotomine sandfly species of the genera Phlebotomus (Old World) and Lutzomyia (New World). Cases of VL along with HIV have also been reported by WHO has an emerging and intricate problem (WHO, 2013).

In India, the disease manifests in two forms, the Cutaneous (dry and wet) and Visceral (kala-azar) variety. The disease may be sporadic, endemic or epidemic. About 500 species of 6 genera of female 'Phlebotomus' are suspected or proven as vectors transmitting parasites from animal to animal, animal to man and man to man. Leishmaniasis is endemic in 98 countries or territories, with more than 350 million people at risk. The global incidence is estimated to be 2 million new cases per year $(0.5$ million of VL and 1.5 million of CL). VL causes an estimated 50,000 deaths annually, a rate surpassed among parasitic diseases only by malaria and 2,357,000 disability-adjusted life years lost, placing leishmaniasis ninth in a global analysis of infectious diseases (WHO, 2010).

Higher altitudes are generally characterized by lower temperatures and a lower relative humidity and these indicators have a tendency of greater fluctuations which affect the distribution of the vector negatively. In the lowlands, on the other hand, humans are especially at risk due to widespread situations suitable for the sand fly vector which prefers a relative humidity of $70-80 \%$ and temperatures between 25 and $28^{\circ} \mathrm{C}$. However, transmission is more focally distributed than in many highland areas since the breeding sites of the vector are concentrated to spatial locations such as river banks, alluvial soil and dark, damp places such as deep cracks in the soil or rock (Sharma and Singh, 2008; Kalluri et al., 2007).

\section{MATERIALS AND METHODS}

This method utilizes retrospective study on visceral leishmaniasis cases, who were admitted to RMRIMS, Patna, India from January 2013 to April, 2013. The data was obtained from hospital patient information system. The following information was obtained for each patient: age, sex and place of residence, date of admission, clinical features on admission and outcomes of cases enrolled in the study. The total number of cases were 133 including 101 males and 32 females. The diagnosis was established on the basis of patient history, clinical examinations and laboratory investigations.

\section{RESULTS}

In this study, it was found that $75.93 \%$ cases were of males, $24.07 \%$ cases were of females and the male to female ratio was $3: 1$. The majority of cases were found to be of patients aged between 15 to 45 years of age i.e., $64.67,21.80 \%$ cases were of children below 14 years of age whereas the least number of cases belong to patients of more than 45 years of age i.e., 13.53\%. (Table 1 and Fig. 1-3) Almost, majority of cases belong to the poor people of rural areas of Bihar, India.

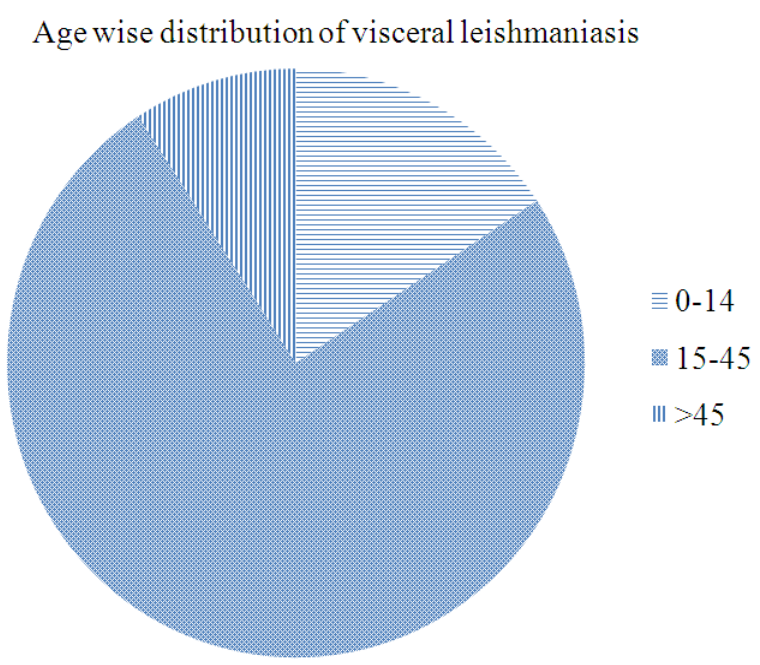

Fig. 1. Pie chart representing cases of different age groups

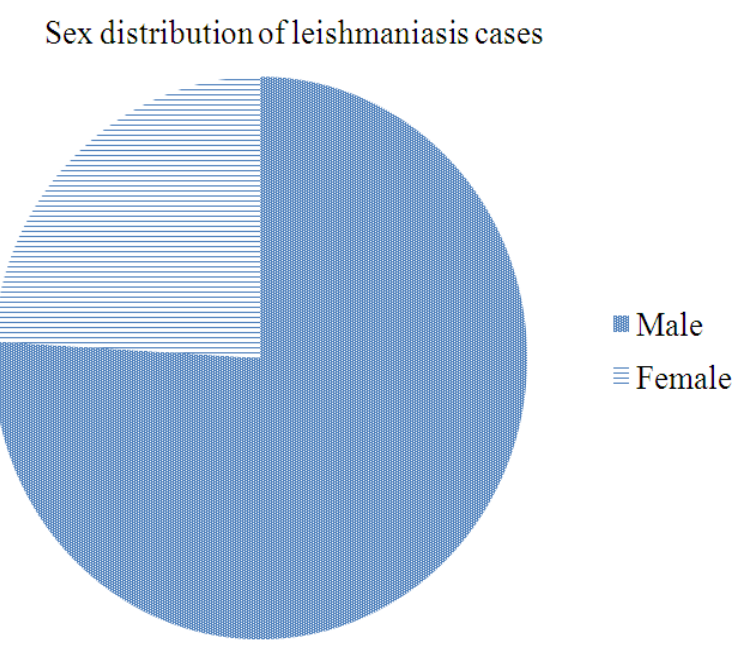

Fig. 2. Pie chart representing sex distribution of cases 


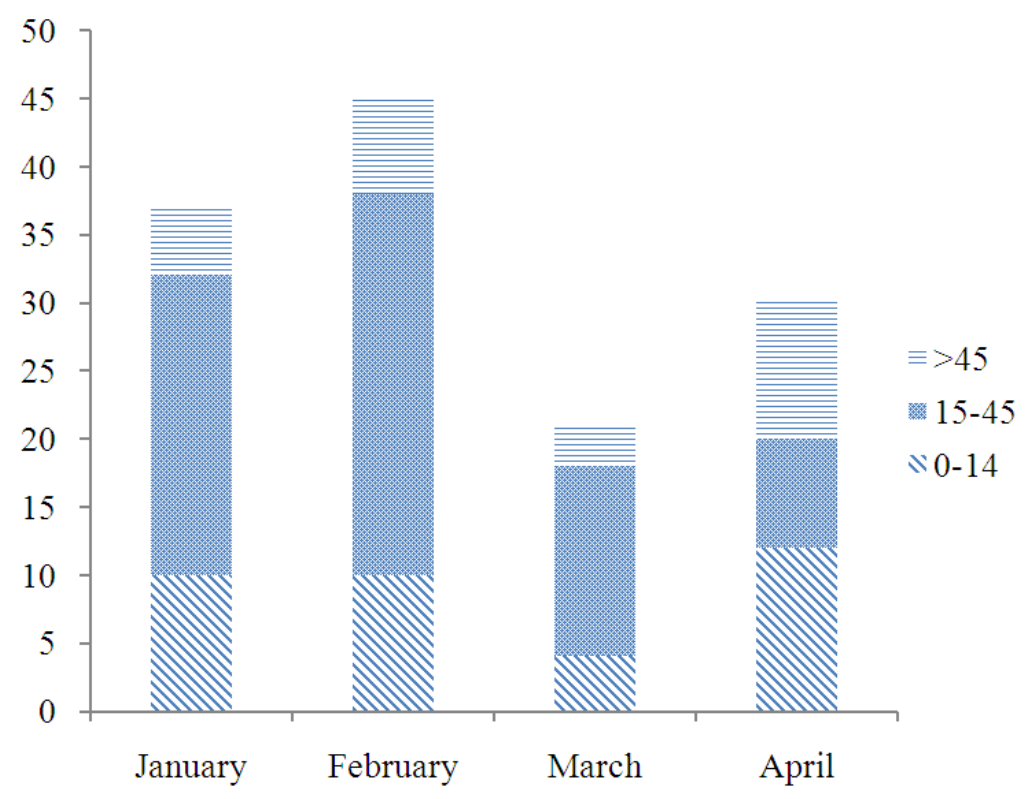

Fig. 3. Bar graph representing different age group in different months

Table 1. Age and sex distribution of visceral leishmaniasis

\begin{tabular}{lllccll}
\hline \multicolumn{5}{c}{} & \multicolumn{5}{c}{ Distribution of cases in different age groups } \\
State & Month & $0-14$ & $15-45$ & $>45$ & Male: Female ratio & Source of data \\
\hline \multirow{4}{*}{ Bihar, India } & January & 10 & 22 & 5 & $1.84: 1$ & RMRIMS, \\
& February & 10 & 28 & 7 & $4: 01$ & Hospital, \\
& March & 4 & 14 & 3 & $4.25: 1$ & Patna, \\
& April & 12 & 8 & 10 & $2.33: 1$ & India \\
\hline
\end{tabular}

\section{DISCUSSION}

The purpose of this study was to find out the epidemiological data of admitted Visceral Leishmaniasis cases in a research hospital in Patna, (Bihar) India, also focus on the risk factors contributing to the high rate of infection in rural communities. Visceral Leishmaniasis (Kala-azar) is also known as poor man's disease because it affects the poorest of the poor. The question arises why the incidence of kalaazar is more in Bihar? Some of the reasons that may be attributable to this disease of poorest of the poor:

- Huge population

- High poverty

- Low socio-economic status

- Impoverished back ground

- High cost of medication
Additional risk factors attributable to the development of kala-azar in Bihar, India may include:

- Poor education status

- History of other diseases in the previous years

- Familial history of kala-azar

- Types of walls (mud plastered walls) in houses

- Presence of granary in houses

- Presence of vegetation around houses

- Bamboo trees near houses

- Irregular insecticide spraying

On the basis of this epidemiological study, it was observed that the incidence of VL is more in males (75.93\%), compared to females $(24.07 \%)$. It is also evident from the study that $15-45$ age group people are more vulnerable to the infection. 


\section{CONCLUSION}

At present Visceral Leishmaniasis (kala-azar) is reemerging serious health problem in our country, especially in Bihar. It is affecting mainly the poorest section of society, affecting men more than women. The cost of treatment is also very high as well as difficult due to development of extensive resistance to the first line therapy. Kala-azar can be prevented and controlled by early detection and prompt treatment of cases along with adoption of preventive measures. The prevention of kalaazar should be given emphasis or the risk of infection can be reduced through health education, patient education and counselling, regular spraying of insecticides such as DDT, as well as individual protective measure such as-avoid sleeping on the floor, repairing cracks and crevices in the floors, walls of mud houses, using fine mesh nets around the bed, use of insect repellent, avoid sleeping in cow shades to avoid bite of sand fly vector, as well as keeping environment hygienic and clean. From above study it is concluded that more better understanding and awareness is required to prevent and eradicate the problem of VL infection from rural communities.

\subsection{Conflict of Results}

There is no conflict of results.

\section{ACKNOWLEDGEMENT}

The researchers feel great thanks and regards to Director, Medical Officers and all other health professionals of RMRIMS, Patna, India for their valuable guidance and support.

\section{REFERENCES}

Kalluri, S., P. Gilrut, D. Rogers and M. Zzczur, 2007. Surveillance of arthropod vector-borne infectious diseases using remote sensing techniques: A review. PLoS Pathog., 3: 1361-1371. PMID: 17967056

Ready, P.D., 2008. Leishmaniasis emergence and climate change. Rev. Sci. Tech., 27: 399-412. PMID: 18819668

Sharma, U. and S. Singh, 2008. Insect vectors of Leishmania: Distribution, physiology and their control. J. Vector Borne Dis., 45: 255-272. PMID: 19248652

WHO, 2008. The Global Burden of Disease: 2004 Update. 1st Edn., World Health Organization, Geneva, Switzerland, ISBN-10: 9241563710, pp: 1464.

WHO, 2010. Control of the Leishmaniasis. WHO, Geneva, pp: 104.

WHO, 2013. Leishmaniasis and HIV coinfection. WHO. 